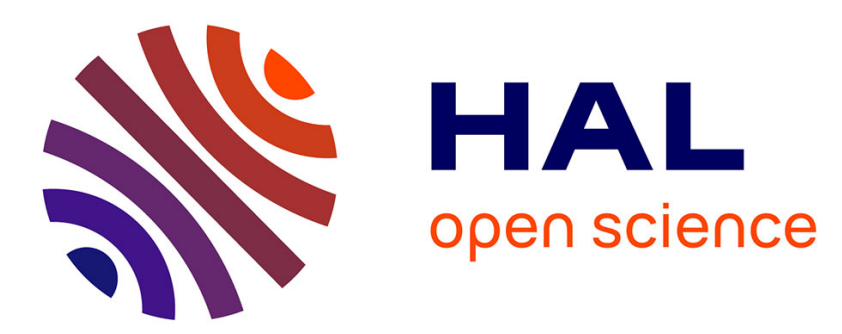

\title{
Polynomial skew-products in dimension 2: Bulging and Wandering Fatou components
}

\author{
Jasmin Raissy
}

\section{To cite this version:}

Jasmin Raissy. Polynomial skew-products in dimension 2: Bulging and Wandering Fatou components. Bollettino dell'Unione Matematica Italiana, 2017, 10 (3), pp.441 - 450. 10.1007/s40574-016-0101-1. hal-01610339

\section{HAL Id: hal-01610339 \\ https://hal.science/hal-01610339}

Submitted on 11 Oct 2017

HAL is a multi-disciplinary open access archive for the deposit and dissemination of scientific research documents, whether they are published or not. The documents may come from teaching and research institutions in France or abroad, or from public or private research centers.
L'archive ouverte pluridisciplinaire HAL, est destinée au dépôt et à la diffusion de documents scientifiques de niveau recherche, publiés ou non, émanant des établissements d'enseignement et de recherche français ou étrangers, des laboratoires publics ou privés. 


\title{
Polynomial skew-products in dimension 2: Bulging and Wandering Fatou Components
}

\author{
Jasmin Raissy
}

Received: date / Accepted: date

\begin{abstract}
In this short note we give an updated account of the recent results on Fatou components for polynomial skew-products in complex dimension two in a neighbourhood of an invariant fiber, dividing our discussion according to the different possible kinds of invariant fibers.
\end{abstract}

Keywords Polynomial skew-products · bulging Fatou components · wandering Fatou components

Mathematics Subject Classification (2000) $32 \mathrm{H} 50 \cdot 37 \mathrm{~F} 10$

\section{Introduction}

Consider the discrete holomorphic dynamical system given by a complex manifold $X$ and the iteration of a holomorphic endomorphim $F: X \rightarrow X$. In the investigation of the global behaviour of such a system it is natural to introduce the Fatou set of $F$, that is the largest open set $\mathscr{F}(F)$ where the family of iterates $\left\{F^{n}\right\}_{n \in \mathbb{N}}$ of $F$ is normal. A connected component of the Fatou set is called a Fatou component.

In complex dimension one, Fatou components of rational maps of degree at least 2 on the Riemann sphere are well understood. In fact, from one hand, Fatou conjectured the following classification for invariant Fatou components, which was partially proven by Fatou himself and completed by several authors (Julia, Leau, Siegel, Herman, Yoccoz...).

Theorem 1 (Fatou's Classification of invariant Fatou components) Let $f: \mathbb{P}^{1}(\mathbb{C}) \rightarrow$ $\mathbb{P}^{1}(\mathbb{C})$ be a rational map of degree $d \geq 2$ on the Riemann sphere. Let $\Omega$ be an invariant Fatou component of $f$. Then $\Omega$ is either:

Partially supported by the ANR project LAMBDA, ANR-13-BS01-0002, and by the FIRB2012 grant "Differential Geometry and Geometric Function Theory", RBFR12W1AQ 002.

\section{J. Raissy}

Institut de Mathématiques de Toulouse; UMR5219

Université Toulouse III - Paul Sabatier

118 route de Narbonne

UPS IMT, F-31062 Toulouse Cedex 9

France

E-mail: jraissy@math.univ-toulouse.fr 
(i) the basin of an attracting fixed point p, i.e. $\left|f^{\prime}(p)\right|<1$,

(ii) the parabolic basin of a parabolic fixed point $p$, i.e. $\left|f^{\prime}(p)\right|$ is a root of unity, and in this case $f^{\prime}(p)=1$,

(iii) a rotation domain, that can be a Siegel disk of an elliptic fixed point $p$, i.e. $f^{\prime}(p)=e^{2 \pi i \theta}$ with $\theta \in \mathbb{R} \backslash \mathbb{Q}$, or a Herman ring.

We recall that a connected open set $U$ is called:

- a basin of an attracting fixed point $p$ if it contains a fixed point $p$ such that $\left|f^{\prime}(p)\right|<1$ and the sequence of iterates $\left\{f^{n}\right\}$ converges uniformly to $p$ on every compact subset of $U$;

- a parabolic basin if there is a fixed point $p \in \partial U$ with $|f(p)|=1$, and the sequence of iterates $\left\{f^{n}\right\}$ converges uniformly to $p$ on every compact subset of $U$;

- a Siegel disk if it is simply connected, and there exists a holomorphic isomorphism $h: U \rightarrow \mathbb{D}$ such that $h \circ f \circ h^{-1}(z)=e^{2 \pi i \theta} z$, with $\theta \in \mathbb{R} \backslash \mathbb{Q}$;

- a Herman ring if it is doubly connected, and there exists a radius $r>0$ and a holomorphic isomorphism $h: U \rightarrow A_{r}:=\{z \in \mathbb{C}|r<| z \mid<1\}$, such that $h \circ f \circ h^{-1}(z)=e^{2 \pi i \theta} z$, with $\theta \in \mathbb{R} \backslash \mathbb{Q}$.

On the other hand, Sullivan proved his celebrated non-wandering domains theorem.

Theorem 2 (Sullivan [30]) Let $f: \mathbb{P}^{1}(\mathbb{C}) \rightarrow \mathbb{P}^{1}(\mathbb{C})$ be a rational map of degree $d \geq 2$. Then every Fatou component of $f$ is (pre-)periodic.

Therefore, up to considering iterates of $f$ we can describe all its Fatou components. The proof of Sullivan's non-wandering Theorem strongly relies on the Ahlfors-Bers mesurable mapping Theorem for quasi-conformal functions. Such a result is strongly one-dimensional and does not have an analogue in higher dimension, making impossible to mimic Sullivan's proof there. Moreover, in complex dimension two, the understanding of Fatou components is far less complete. A considerable progress in the classification of periodic Fatou components has been achieved thanks to Bedford and Smillie [2] [3] [4], Fornæss and Sibony [13], Lyubich and Peters [21] and Ueda [31].

The question of the existence of wandering (i.e., not pre-periodic) Fatou components in higher dimension was put forward by several authors since the 1990's (see e.g. [15]). Higher dimensional transcendental mappings with wandering domains can be constructed from one-dimensional examples by taking direct products. An example of a transcendental biholomorphism of $\mathbb{C}^{2}$ with a wandering Fatou component oscillating to infinity was constructed by Fornæss and Sibony in [14]. Nonetheless, until recently very little was known about the existence of wandering Fatou components for holomorphic endomorphisms of $\mathbb{P}^{2}(\mathbb{C})$ or for polynomial endomorphisms of $\mathbb{C}^{2}$.

A first natural class of maps to consider are direct product polynomial endomorphisms of $\mathbb{C}^{2}$, that is maps $F: \mathbb{C}^{2} \rightarrow \mathbb{C}^{2}$ of the form

$$
F(z, w)=(f(z), g(w)),
$$

where $f$ and $g$ are complex polynomials in one variable. This allows us to recover the generalizations of one-dimensional dynamical behaviours in dimension two, but without giving us a complete understanding of all possible behaviours of polynomial endomorphisms in $\mathbb{C}^{2}$, as direct products are very particular cases.

A more interesting class to consider is given by polynomial skew-products in $\mathbb{C}^{2}$, namely polynomial maps $F: \mathbb{C}^{2} \rightarrow \mathbb{C}^{2}$ of the form

$$
F(z, w)=(f(z, w), g(w)),
$$


where $g$ is a complex polynomial in one variable and $f$ is a complex polynomial in two variables. Since they preserve the fibration $\{w=$ const. $\}$, skew-products allow us to build on one-dimensional dynamics and to get a first flavor of the richness of the higher dimension setting we are working in. This idea has been used by several authors to construct maps with particular dynamical properties. Dujardin, for example, used in [11] specific skew-products to construct a non-laminar Green current. Boc-Thaler, Fornæss and Peters constructed in [6] a map having a Fatou component with a punctured limit set. Last but not least, as we shall recall in section 3 skew-products are one of the key ingredients in the construction obtained very recently by Astorg, Buff, Dujardin, Peters and the author [1] of holomorphic endomorphisms of $\mathbb{P}^{2}(\mathbb{C})$ having a wandering Fatou component.

The investigation of the holomorphic dynamics of polynomial skew-products was started by Heinemann [16] and then continued by Jonsson [18]. The topology of Fatou components of skew-products has been studied by Roeder in [26].

Given a Fatou component $\Omega$ of a polynomial skew-product $F$ in $\mathbb{C}^{2}$, its projection on the second coordinate $\Omega_{2}=\pi_{2}(\Omega)$ is a Fatou components for $g$ and hence thanks to Sullivan's non-wandering Theorem 2 up to considering an iterate of $F$, it has to fall into one of the three cases given by Theorem 1 and moreover, since we are considering polynomials, Herman rings cannot occur. Therefore, since fixed points for $g$ correspond to invariant fibers for $F$, up to considering an iterate of $F$, we can restrict ourselves to study what happens in neighbourhoods of invariant fibers of the form $\{w=c\}$. One-dimensional theory also describes the dynamics on the invariant fiber, which is given by the action of the one-dimensional polynomial $f(c, z):=f_{c}(z)$, and hence the Fatou components of $f_{c}$ will be again all preperiodic and, up to consider an iterate, we can assume that they are either attracting basins, or parabolic basins or Siegel disks. This structure leads us to two immediate questions.

- Question 1. Do all Fatou components of $f_{c}$ bulge to two-dimensional Fatou components of $F$ ?

- Question 2. Is it possible to have wandering Fatou components for $F$ in a neighbourhood of an invariant fiber?

In the following we shall call an invariant fiber $\{w=c\}$ attracting, parabolic or elliptic according to whether $c$ is an attracting, parabolic or elliptic fixed point for $g$. A bulging Fatou component will be a Fatou component $\Omega$ of $F$ such that $\Omega \cap\{w=c\}$ is a one-dimensional Fatou component of $f_{c}$ on the invariant fiber $\{w=c\}$. With a slight abuse of terminology we shall say that a Fatou component $\Omega_{c}$ of $f_{c}$ on the invariant fiber $\{w=c\}$ is bulging if there exists a bulging Fatou component $\Omega$ of $F$ so that $\Omega_{c}=\Omega \cap\{w=c\}$.

The purpose of this note is to provide an updated account of the results related to these questions. We shall divide our discussion according to the different possible kinds of invariant fibers.

\section{Attracting invariant fiber}

Let us consider a polynomial skew-product $F: \mathbb{C}^{2} \rightarrow \mathbb{C}^{2}$ of degree $d \geq 2$

$$
F(z, w)=(f(z, w), g(w))
$$

with an attracting invariant fiber. We can assume without loss of generality that the invariant fiber is $\{w=0\}$. Therefore we have $g(0)=0$ and $\left|g^{\prime}(0)\right|<1$. In this case it is a wellknown one-dimensional result (see for exemple [9] or [22]) that there exists an attracting 
basin, containing the origin, of points whose iterates converge to the origin. The rate of convergence to the fixed point depends on whether $g^{\prime}(0)=0$, in which case the fixed point is called superattracting, or $g^{\prime}(0) \neq 0$, in which case the fixed point is called attracting or geometrically attracting.

\subsection{Superattracting case}

This setting was studied by Lilov in [20] who was able to answer both questions stated in the introduction. He first proved the following result giving a positive answer to Question 1.

Theorem 3 (Lilov [20]) Let $F: \mathbb{C}^{2} \rightarrow \mathbb{C}^{2}$ be a polynomial skew-product of the form (1) of degree $d \geq 2$. Let $\{w=c\}$ be a superattracting invariant fiber for $F$. Then all onedimensional Fatou components of $f_{c}$ bulge to Fatou components of $F$.

Idea of the proof. We can assume $c=0$ without loss of generality. Thanks to Theorem 1 and Theorem 2, all Fatou components of the restriction $f_{0}(z)=f(z, 0)$ of $f(z, w)$ to the invariant fiber are (pre-)periodic and are either attracting basins, or parabolic basins or Siegel domains. The strategy of the proof is to prove separately for each of these cases that the corresponding component is contained in a two-dimensional Fatou component of $F$. The bulging of one-dimensional Fatou components of attracting periodic points of $f_{0}(z)$ is wellknown and follows for instance from the results of Rosay and Rudin [27]. For the remaining cases, by [20, Theorem 3.17] there exists a strong stable manifold through all point in the one-dimensional Fatou components of parabolic or elliptic periodic points of $f_{0}(z)$, and so the corresponding bulging Fatou components simply consist of the union of such manifolds.

Then Lilov proved the following result implying the non-existence of wandering Fatou components in a neighbourhood of a superattracting invariant fiber.

Theorem 4 (Lilov [20]) Let $F: \mathbb{C}^{2} \rightarrow \mathbb{C}^{2}$ be a polynomial skew-product of the form (1) of degree $d \geq 2$. Let $\{w=c\}$ be a superattracting invariant fiber for $F$ and let $\mathscr{B}$ be the immediate basin of the superattracting fixed point $c$. Let $w_{0} \in \mathscr{B}$ and let $D_{w_{0}}$ be a onedimensional open disk lying in the fiber over $w_{0}\left(\mathbb{C} \times\left\{w_{0}\right\}\right)$. Then the forward orbit of $D$ must intersect one of the bulging Fatou components of $f_{c}$.

The proof relies on the repeated use of [20, Lemma 3.2.4] to the orbit of a disk lying in a fiber over a point in the attracting basin, in order to obtain estimates from below for the radii of the images. Thanks to [20, Proposition 3.2.8], by studying the geometry of the bulging Fatou components, it is also possible to obtain an upper bound on the largest possible disk lying in a fiber over a point in the attracting basin that can lie in the complement of a bulging Fatou component, depending on the distance to the invariant fiber. The conclusion then follows combining these two estimates.

All bulging Fatou components are (pre-)periodic, therefore all Fatou components for $F$ in a neighbourhood of a superattracting invariant fiber are (pre-)periodic, and then the nonexistence of wandering Fatou components in a neighbourhood of a superattracting invariant fiber follows immediately.

Corollary 1 (Lilov [20]) Let $F: \mathbb{C}^{2} \rightarrow \mathbb{C}^{2}$ be a polynomial skew-product of the form (1) of degree $d \geq 2$. Let $\{w=c\}$ be a superattracting invariant fiber for $F$ and let $\mathscr{B}$ be the immediate basin of the superattracting fixed point $c$. Then there are no wandering Fatou components in $\mathscr{B} \times \mathbb{C}$. 
2.2 Geometrically attracting case

The geometrically attracting case was first partially addressed by Lilov in [20] even if not stated explicitly. In fact, the proof of Theorem 3 can be readily adjusted to this case obtaining the following statement answering Question 1.

Theorem 5 (Lilov [20]) Let $F: \mathbb{C}^{2} \rightarrow \mathbb{C}^{2}$ be a polynomial skew-product of the form (1) of degree $d \geq 2$. Let $\{w=c\}$ be an attracting invariant fiber for $F$, that is $\left|g^{\prime}(c)\right|<1$. Then all one-dimensional Fatou components of $f_{c}$ bulge to Fatou components of $F$.

On the other hand, the proof of Theorem 4 cannot be generalized to this setting, which is indeed more complicated than the superattracting case. In fact, Theorem 4 does not hold in general, as showed by Peters and Vivas with the following result.

Theorem 6 (Peters-Vivas, [25]) Let $F: \mathbb{C}^{2} \rightarrow \mathbb{C}^{2}$ be a polynomial skew-product of the form

$$
F(z, w)=(p(z)+q(w), \lambda w)
$$

with $0<|\lambda|<1$ and $p$ and $q$ complex polynomials. Then there exists a triple $(\lambda, p, q)$ and $a$ holomorphic disk $D \subset\left\{w=w_{0}\right\}$ whose forward orbit accumulates at a point $\left(z_{0}, 0\right)$, where $z_{0}$ is a repelling fixed point in the Julia set of $f_{0}$.

As a consequence, the forward orbits of $D$ cannot intersect the bulging Fatou components of $f_{0}$. The family $\left\{\left.F\right|_{D} ^{n}\right\}_{n \in \mathbb{N}}$ is normal on the disks $D$, and so these are Fatou disks. However such disks are completely contained in the Julia set of $F$, which is the complement in $\mathbb{C}^{2}$ of the Fatou (see [25, Theorem 6.1]).

The geometrically attracting case have been further investigated by Peters and Smit in [24]. They focused their investigation on polynomial skew-products such that the action on the invariant attracting fiber is subhyperbolic, that is the polynomial does not have parabolic periodic points and all critical points lying on the Julia set are pre-periodic. They proved the following result.

Proposition 1 (Peters-Smit, [24]) Let $F: \mathbb{C}^{2} \rightarrow \mathbb{C}^{2}$ be a polynomial skew-product of the form (1). Assume that the origin is an attracting, not superattracting, fixed point for $g$ with corresponding basin $B_{g}$, and the polynomial $f_{0}(z):=f(z, 0)$ is subhyperbolic. Then there exists a set $E \subset \mathbb{C}$ of full mesure, such that for every $w_{0} \in E$ the forward orbit of every disk in the fiber $\left\{w=w_{0}\right\}$ must intersect a bulging Fatou component of $f_{0}$.

Idea of the proof. Notice that it suffices to prove the proposition in a neighbourhood of the attracting fiber $\{w=0\}$. Therefore, up to considering a smaller neighbourhood, we can assume without loss of generality that $g(w)=\lambda w$, and

$$
f(z, w)=a_{0}(w)+a_{1}(w) z+\cdots+a_{d}(w) z^{d}
$$

where $a_{0}(w), \ldots, a_{d}(w)$ are holomorphic functions in $w$. The subhyperbolicity of the polynomial $f_{0}$ implies that its Fatou set is the union of finitely many attracting basins, and the orbits of the critical points contained in the Fatou set converge to one of these attracting cycles. The proof can be divided into 5 main steps.

Step 1. Fix $R>0$ large enough so that for all $z$ such that $|z|>R$ we have $\left|f_{0}(z)\right|>2|z|$ and set

$$
W_{0}=\{|z|>R\} \cup \bigcup_{y \in \operatorname{Att}\left(f_{0}\right)} W_{y}
$$


where $\operatorname{Att}\left(f_{0}\right)$ is the set of all attracting periodic points of $f_{0}$, and for each $y \in \operatorname{Att}\left(f_{0}\right)$ the set $W_{y}$ is an open neighbourhood of the orbit of $y$ such that $\overline{f_{0}\left(W_{y}\right)} \subset W_{y}$. Fix a neighbourhood $U$ of the post-critical set of $f_{0}$. Then by [24, Proposition 15], there exists a set $E \subset \mathbb{C}$ of full mesure in a neighbourhood of the origin such that for all $w_{0} \in E$ there exists a constant $C=C\left(w_{0}, U\right)$ such that for all $n \in \mathbb{N}$ we have

$$
\text { Card }\left\{z: \frac{\partial F_{1}^{n}}{\partial z}\left(z, w_{0}\right)=0 \text { and } F_{1}^{n}\left(z, w_{0}\right) \notin W_{0} \times U\right\} \leq C \sqrt{n},
$$

where $F_{1}^{n}$ is the first component of the $n$-th iterate of $F$.

Step 2. Assume by contradiction that a fiber $\left\{w=w_{0}\right\}$, with $w_{0} \in E$, contains a disk $D$ whose forward orbit avoids the bulging Fatou components of $f_{0}$. Then the restriction of $F^{n}$ to $D$ is bounded and hence a normal family. Therefore, up to shrinking $D$ there exists a subsequence $F^{n_{j}}$ such that $\left.F^{n_{j}}\right|_{D}$ converges, uniformly on compact subsets of $D$, to a point $\zeta$ in the Julia set of $f_{0}$. Moreover, there exists $\varepsilon>0$ so that $F^{n}(D) \cap\left(W_{0} \times D(0, \varepsilon)\right)$ is empty for all $n \in \mathbb{N}$.

Step 3. Each critical point $x$ contained in the Julia set is eventually mapped into a repelling periodic point, and up to considering an iterate of $F$ we may assume that it is eventually mapped into a repelling fixed point with multiplier $\mu$, with $|\mu|>1$. The main tool to control the orbits of the critical points of $F$ is obtained using a linearization map of the unstable manifold of the repelling fixed point, given by a map $\Phi: \mathbb{C} \rightarrow \mathbb{C}$ satisfying $\Phi(\mu t)=f_{0}^{k}$ 。 $\Phi(t)$ for some $k \in \mathbb{N}$. Thanks to [24, Proposition 10], there exist $\widetilde{C}>1$ and $0<\gamma<1$ so that

$$
\operatorname{Area}\left(F^{n}(D)\right) \leq \widetilde{C} \gamma^{n}
$$

Step 4. We may assume that $\zeta$ does not lie in the post-critical set, and we may choose $U$ and $r>0$ such that $D(\zeta, r) \cap\left(U \cup W_{0}\right)=\emptyset$. Let $j_{1} \in \mathbb{N}$ be such that $F_{1}^{n_{j}}(D) \subseteq D\left(\zeta, \frac{r}{2}\right)$ for all $j \geq j_{1}$, and consider $O_{j}$ the connected component of $\left(F^{n_{j}}\right)^{-1}\left(D(\zeta, r) \times\left\{\lambda^{n_{j}} w_{0}\right\}\right)$ containing $D$. Then $D \subseteq O_{j} \subseteq D(0, R) \times\left\{w_{0}\right\}$, and we can study the proper holomorphic function $F_{1}^{n_{j}}: O_{j} \rightarrow D(\zeta, r)$. Thanks to 3 , such a map has at most $d_{j}=C \sqrt{n_{j}}$ critical points.

Step 5. It is possible (see [24, Proposition 28]) to find a uniform constant $C_{1}>0$ so that if $f: \mathbb{D} \rightarrow \mathbb{D}$ is a proper holomorphic function of degree $d$, the set $R \subset \mathbb{D}$ has Poincare area equal to $A$, and $d \cdot A^{1 / 2 d}<8$, then the Poincaré area of $f^{-1}(R)$ is at most $C_{1} d^{3} A^{1 / d}$. Then, setting $R_{j}=F_{1}^{n_{j}}(D)$ and denoting by $A_{j}$ its Poincaré area $\operatorname{Area}_{D(\zeta, r)}\left(R_{j}\right)$ with respect to $D(\zeta, r)$, for $j \geq j_{1}$, we have $R_{j} \subseteq D(\zeta, r)$, and we can estimate $A_{j}$ applying (4). Therefore there exists $j_{2} \geq j_{1}$ such that $d_{j} A_{j}^{1 / 2 d_{j}}<1 / 8$ for all $j \geq j_{2}$. This implies

$$
\operatorname{Area}_{D(0, R)}(D) \leq \operatorname{Area}_{O_{j}}(D) \leq C_{2} d_{j}^{3} A_{j}^{1 / d_{j}} \leq M n_{j}^{3 / 2} \gamma^{n_{j}^{3 / 2}}
$$

where $M>0$. The contradiction follows from the fact that the last expression will converge to zero as $j$ increases towards infinity.

Thanks to the fact that in particular $E$ is dense, Peters and Smit are able to give a negative answer to Question 2 when the action on the invariant fiber is subhyperbolic. They also obtain as a corollary that the only Fatou components of $F$ are the bulging ones, since the topological degree of $F$ equals the one of $f_{0}$, implying that the only Fatou components that can be mapped onto the bulging Fatou components of $f_{0}$ are exactly those bulging Fatou components. 
Theorem 7 (Peters-Smit, [24]) Let $F: \mathbb{C}^{2} \rightarrow \mathbb{C}^{2}$ be a polynomial skew-product of the form (1). Assume that the origin is an attracting fixed point for $g$ with corresponding basin $B_{g}$, and the polynomial $f_{0}(z):=f(z, 0)$ is subhyperbolic. Then $F$ has no wandering Fatou component over $B_{g}$.

\section{Parabolic invariant fiber}

A first contribution to the investigation of this case is due to Vivas, who proved a parametrization result [32, Theorem 3.1] for the unstable manifolds for special parabolic skew-product of $\mathbb{C}^{2}$. Vivas used this parametrization as the main tool to prove the analogue of Theorem 7 for special parabolic skew-product. However, also this construction does not allow to construct a wandering Fatou component in a neighbourhood of the parabolic invariant fiber.

The existence of polynomial skew-products of $\mathbb{C}^{2}$, extending to holomorphic endomorphisms of $\mathbb{P}^{2}(\mathbb{C})$, having a wandering Fatou component has been proved in [1] by Astorg, Buff, Dujardin, Peters and the author. The idea of using parabolic implosion techniques on polynomial skew products was suggested by Lyubich. The main strategy is to combine slow convergence to an invariant parabolic fiber and parabolic transition in the fiber direction, to produce orbits shadowing those of the so-called Lavaurs map.

Theorem 8 (Astorg-Buff-Dujardin-Peters-R. [1]) There exists a holomorphic endomorphism $F: \mathbb{P}^{2}(\mathbb{C}) \rightarrow \mathbb{P}^{2}(\mathbb{C})$, induced by a polynomial skew-product mapping $F: \mathbb{C}^{2} \rightarrow \mathbb{C}^{2}$, having a wandering Fatou component. More precisely, let $f: \mathbb{C} \rightarrow \mathbb{C}$ and $g: \mathbb{C} \rightarrow \mathbb{C}$ be polynomials of the form

$$
f(z)=z+z^{2}+\mathrm{O}\left(z^{3}\right) \text { and } g(w)=w-w^{2}+\mathrm{O}\left(w^{3}\right) .
$$

If the Lavaurs map $\mathscr{L}_{f}: \mathscr{B}_{f} \rightarrow \mathbb{C}$ has an attracting fixed point, then the skew-product $F:: \mathbb{C}^{2} \rightarrow \mathbb{C}^{2}$ defined by

$$
F(z, w):=\left(f(z)+\frac{\pi^{2}}{4} w, g(w)\right)
$$

has a wandering Fatou component.

The orbits in these wandering Fatou components are bounded and the approach used in the proof is essentially local. Notice that if $f$ and $g$ have the same degree, $F$ extends to a holomorphic endomorphism of $\mathbb{P}^{2}(\mathbb{C})$. Moreover we can obtain examples in arbitrary dimension $k \geq 2$ by simply considering products mappings of the form $(F, Q)$, where $Q$ has a fixed Fatou component.

To give the definition of Lavaurs map and the main ideas of the proof, we have to recall some facts on parabolic dynamics (more details can be found in [1, Appendix A]). Let $f$ be a polynomial of the form

$$
f(z)=z+z^{2}+a z^{3}+\mathrm{O}\left(z^{4}\right) \text { for some } a \in \mathbb{C} .
$$

and denote by

$$
\mathscr{B}_{f}:=\left\{z \in \mathbb{C} ; f^{n}(z) \underset{n \rightarrow+\infty}{\stackrel{\neq}{\longrightarrow}} 0\right\}
$$


the parabolic basin of 0 . It is a well-known one-dimensional result the existence of an attracting Fatou coordinate $\varphi_{f}: \mathscr{B}_{f} \rightarrow \mathbb{C}$ conjugating $f$ to the translation $T_{1}$ by 1 :

$$
\varphi_{f} \circ f=T_{1} \circ \varphi_{f} .
$$

The Fatou coordinate can be normalized by requiring that

$$
\varphi_{f}(z)=-\frac{1}{z}-(1-a) \log \left(-\frac{1}{z}\right)+o(1) \text { as } \operatorname{Re}\left(-\frac{1}{z}\right) \rightarrow+\infty
$$

where the branch of log used in this normalization, as well as in the next one, is the one defined in $\mathbb{C} \backslash \mathbb{R}^{-}$which vanishes at 1 . There also exists a repelling Fatou parameterization $\psi_{f}: \mathbb{C} \rightarrow \mathbb{C}$ satisfying

$$
\psi_{f} \circ T_{1}=f \circ \psi_{f},
$$

which may be normalized by requiring that

$$
-\frac{1}{\psi_{f}(Z)}=Z+(1-a) \log (-Z)+o(1) \text { as } \operatorname{Re}(Z) \rightarrow-\infty .
$$

The Lavaurs map $\mathscr{L}_{f}$ is then defined by

$$
\mathscr{L}_{f}:=\psi_{f} \circ \varphi_{f}: \mathscr{B}_{f} \rightarrow \mathbb{C}
$$

This kind of functions appear in considering high iterates of small perturbations of $f$ : this phenomenon is known as parabolic implosion, and plays a key role in our construction. A first introduction to this topic can be found in [10], and we also refer to [28] for a very detailed presentation. (Semi-)parabolic implosion was recently studied for dissipative polynomial automorphisms of $\mathbb{C}^{2}$ by Bedford, Smillie and Ueda in [5] (see also [12]).

The strategy of the proof of Theorem 8 is the following. Consider $\mathscr{B}_{g}$ the parabolic basin of 0 under iteration of $g$. For all $w \in \mathscr{B}_{g}$, the orbit $g^{m}(w)$ converges to 0 like $1 / m$. One of the key points is to choose $\left(z_{0}, w_{0}\right) \in \mathscr{B}_{f} \times \mathscr{B}_{g}$ so that the first coordinate of $F^{m}\left(z_{0}, w_{0}\right)$ returns infinitely many times close to the attracting fixed point of $\mathscr{L}_{f}$. The proof is designed so that the return times are the integers $n^{2}$ for $n \geq n_{0}$. Therefore, we need to analyze the orbit segment between $n^{2}$ and $(n+1)^{2}$, which is of length $2 n+1$.

For large $n$, the first coordinate of $F$ along this orbit segment is approximately

$$
f(z)+\varepsilon^{2} \text { with } \frac{\pi}{\varepsilon}=2 n .
$$

Lavaurs Theorem from parabolic implosion states that if $\frac{\pi}{\varepsilon}=2 n$, then for large $n$, the $(2 n)^{\text {th }}$ iterate of $f(z)+\varepsilon^{2}$ is approximately equal to $\mathscr{L}_{f}(z)$ on $\mathscr{B}_{f}$.

Our setting is different since in our case $\varepsilon$ keeps decreasing along the orbit. Indeed on the first coordinate we are taking the composition of $2 n+1$ transformations of the form

$$
f(z)+\varepsilon_{k}^{2} \text { with } \frac{\pi}{\varepsilon_{k}} \simeq 2 n+\frac{k}{n} \text { and } 1 \leq k \leq 2 n+1 .
$$

The key step in the proof of Theorem 8 consists in a detailed analysis of this non-autonomous situation, proving that the decay of $\varepsilon_{k}$ is counterbalanced by taking exactly one additional iterate of $F$. The precise statement is the following. 
Proposition 2 (Astorg-Buff-Dujardin-Peters-R. [1]) As $n \rightarrow+\infty$, the sequence of maps

$$
\mathbb{C}^{2} \ni(z, w) \mapsto F^{2 n+1}\left(z, g^{n^{2}}(w)\right) \in \mathbb{C}^{2}
$$

converges locally uniformly in $\mathscr{B}_{f} \times \mathscr{B}_{g}$ to the map

$$
\mathscr{B}_{f} \times \mathscr{B}_{g} \ni(z, w) \mapsto\left(\mathscr{L}_{f}(z), 0\right) \in \mathbb{C} \times\{0\} .
$$

With this proposition in hand, the proof of the Theorem 8 is easily completed. In fact, let $\xi$ be an attracting fixed point of $\mathscr{L}_{f}$ and let $\left(z_{0}, w_{0}\right)$ be a point in $\mathscr{B}_{f} \times \mathscr{B}_{g}$ chosen so that $F^{n_{0}^{2}}\left(z_{0}, w_{0}\right)$ is close to $(\xi, 0)$ for some large $n_{0}$. Then $F^{\left(n_{0}+1\right)^{2}}\left(z_{0}, w_{0}\right)$ gets closer to $(\xi, 0)$ and we can repeat the process to obtain that the sequence $\left(F^{n^{2}}\left(z_{0}, w_{0}\right)\right)_{n \geq 0}$ converges to $(\xi, 0)$. This argument holds on an open set of initial conditions, therefore these points belong to a Fatou component $\Omega$. For any integer $j \geq 0$, the sequence of maps $\left\{F^{n^{2}+j}\right\}_{n \in \mathbb{N}}$ converges locally uniformly to $F^{j}(\xi, 0)=\left(f^{j}(\xi), 0\right)$ on $\Omega$. Therefore the sequence $\left\{F^{n^{2}}\right\}_{n \in \mathbb{N}}$ converges locally uniformly to $\left(f^{j}(\xi), 0\right)$ on $F^{j}(\Omega)$. If $i, j$ are nonnegative integers such that $F^{i}(\Omega)=F^{j}(\Omega)$, then $f^{i}(\xi)=f^{j}(\xi)$, and so $i=j$ because $\xi$ cannot be pre-periodic under iteration of $f$, since it belongs to the parabolic basin $\mathscr{B}_{f}$. This proves that $\Omega$ is not (pre-)periodic under iteration of $F$, and so it is a wandering Fatou component for $F$.

We end this section recalling some explicit examples satisfying the assumption of Theorem 8 .

Example 1 ([1, Proposition B]) Let $f: \mathbb{C} \rightarrow \mathbb{C}$ be the cubic polynomial $f(z)=z+z^{2}+a z^{3}$, and $g$ be as in (5). If $r>0$ is sufficiently small and $a \in D(1-r, r)$, then the polynomial skew-product $F$ defined in (6) admits a wandering Fatou component.

It is also interesting to search for real polynomial mappings with wandering Fatou domains intersecting $\mathbb{R}^{2}$. We also have such examples.

Example 2 ([1, Proposition $\mathrm{C}]$ ) Let $f: \mathbb{C} \rightarrow \mathbb{C}$ be the degree 4 polynomial defined by

$$
f(z):=z+z^{2}+b z^{4} \text { with } b \in \mathbb{R} .
$$

There exist parameters $b \in(-8 / 27,0)$ such that for $g$ as in $(5)$, the polynomial skew-product $F$ defined in (6) has a wandering Fatou component intersecting $\mathbb{R}^{2}$.

\section{Elliptic invariant fiber}

In a joint project with Peters, we investigated the case of invariant fibers at the center of a Siegel disk. In [23] we prove that all attracting and parabolic Fatou components of a polynomial skew-product with an elliptic invariant fiber with Brjuno rotation number bulge. This allows us to prove that if the rotation number of the elliptic invariant fiber is Brjuno and all critical points of the polynomial acting on the invariant fiber lie in basins of attracting or parabolic cycles, then there are no wandering Fatou components in a neighbourhood of the invariant fiber.

Describing the general situation seems to be more complicated as there might be resonance phenomena. For example, an invariant fiber at the center of a Siegel disk was used in [6] to construct a non-recurrent Fatou component with limit set isomorphic to a punctured 
disk, and in their construction the invariant fiber also contains a Siegel disk, but with opposite rotation number. Moreover, it might happen that Fatou components on the invariant fiber do not bulge. For example, we can consider the skew-product

$$
F(z, w)=\left(\lambda z(1+a z w), \lambda^{-1} w\right)
$$

with $a \in \mathbb{C}^{*}, \lambda=e^{2 \pi i \theta}$ and $\theta \in \mathbb{R} \backslash \mathbb{Q}$. We have $F(z, 0)=(\lambda z, 0)$, but the Siegel disk around the origin in $\{w=0\}$ is not bulging, and in fact it follows from [7] and [8] that there exists a Fatou component of parabolic type having on its boundary the origin of $\mathbb{C}^{2}$, which is fixed by $F$.

\section{References}

1. M. Astorg, X. Buff, R. Dujardin, H. Peters, J. Raissy, A two-dimensional polynomial mapping with a wandering Fatou component, Ann. of Math. (2) 184 no. 1, 263-313 (2016) http://dx.doi.org/10.4007/annals.2016.184.1.2

2. E. Bedford, J. SMiLlie, Polynomial diffeomorphisms of $\mathbb{C}^{2}$ : currents, equilibrium measure and hyperbolicity, Invent. Math. 103 (1), 69-99 (1991)

3. E. Bedford, J. Smillie, Polynomial diffeomorphisms of $\mathbb{C}^{2}$ : II. Stable manifolds and recurrence, J. Am. Math. Soc. 4 (4), 657-679 (1991)

4. E. Bedford, J. SMillie, External rays in the dynamics of polynomial automorphisms of $\mathbb{C}^{2}$, In Contemporary Mathematics, vol. 222, 41-79. AMS, Providence, RI (1999)

5. E. Bedford, J. Smillie, T. UedA. Parabolic bifurcations in complex dimension 2. Preprint 2012, arXiv: 1208.2577

6. L. Boc-Thaler, J.E. FornÆss, H. Peters, Fatou components with punctured limit sets, Ergod. Theory Dyn. Syst. 35, 1380-1393 (2015)

7. F. BRACCI, D. ZAITSEV, Dynamics of one-resonant biholomorphisms, J. Eur. Math. Soc. (JEMS) 15 no. 1, 179-200 (2013)

8. F. BRACCI, J. RAISSY, D. ZAITSEV, Dynamics of multi-resonant biholomorphisms, Int. Math. Res. Not. IMRN, no. 20, 4772-4797 (2013)

9. L. Carleson, T.W. Gamelin, Complex dynamics, Universitext: Tracts in Mathematics. SpringerVerlag, New York (1993)

10. A. DouAdy, Does a Julia set depend continuously on the polynomial? Complex dynamical systems (Cincinnati, OH, 1994), 91-138, Proc. Sympos. Appl. Math., 49, Amer. Math. Soc., Providence, RI (1994)

11. R DUJARDIN A non-laminar dynamical Green current, Math.Ann. 365 no. 1-2, 77-91 (2016)

12. R. DUJARDIn, M. LyUBICH, Stability and bifurcations for dissipative polynomial automorphisms of $\mathbb{C}^{2}$. Invent. Math. 200 no. 2, 439-511 (2015)

13. J.E. Fornæss, N. SibONy, Classification of recurrent domains for some holomorphic maps, Math. Ann. 301 (4), 813-820 (1995)

14. J.E. FornæSs, N. SibOny, Fatou and Julia sets for entire mappings in $\mathbb{C}^{k}$. Math. Ann. 311 no. 1 , 27-40 (1998)

15. J.E. FORNÆSS, N. SIBONY, Some open problems in higher dimensional complex analysis and complex dynamics. Publ. Mat. 45 no. 2, 529-547 (2001)

16. S.-M. Heinemann, Julia sets of skew-products in $\mathbb{C}^{2}$, Kyushu J. Math.52 (2), 299-329 (1998)

17. J.H. HubBARD, Parametrizing unstable and very unstable manifolds, Mosc. Math. J. 5 (1), 105-124 (2005)

18. M. Jonsson, Dynamics of polynomial skew-products on $\mathbb{C}^{2}$, Math. Ann.314, 403-447 (1999)

19. G. KenIGS, Recherches sur les intégrales de certaines équations fonctionelles, Ann. Sci. École Norm. Sup. Paris (3e ser.) 1, 1-41 (1884)

20. K. LiLOv, Fatou Theory in Two Dimensions, Ph.D.thesis, University of Michigan (2004) 
21. M. Lyubich, H. Peters, Classification of invariant Fatou components for dissipative Hénon maps, Geom. Funct. Anal. 24, 887-915 (2014)

22. J. Milnor, Dynamics in one complex variable. Third edition. Annals of Mathematics Studies, 160. Princeton University Press, Princeton, NJ (2006)

23. H. Peters, J. RAISSY, Fatou components of polynomial elliptic skew products, preprint 2016, arXiv:1608.08803

24. H. PETERS, I.M. SMIT, Fatou components of attracting skew products, preprint 2015, arXiv:1508.06605

25. H. Peters, L. VIVAs, Polynomial skew-products with wandering Fatou-disks, Math. Z. 283 no. 1-2, 349-366 (2016)

26. R. Roeder, A dichotomy for Fatou components of polynomial skew-products, Conform. Geom. Dyn. 15, 7-19 (2011)

27. J.P. RosAY, W. RUdin, Holomorphic maps from $\mathbb{C}^{n}$ to $\mathbb{C}^{n}$, Trans. Am. Math. Soc. 310(1), 47-86 (1988)

28. M. SHISHIKURA, Bifurcation of parabolic fixed points. The Mandelbrot set, theme and variations, 325363, London Math. Soc. Lecture Note Ser., 274, Cambridge Univ. Press, Cambridge, (2000)

29. S. Sternberg, Local contractions and a theorem of Poincaré, Am. J. Math.79, 809-824 (1957)

30. D. Sullivan, Quasiconformal Homeomorphisms and Dynamics I. Solution of the Fatou-Julia Problem on Wandering Domains, Ann. of Math. (2) 122 no. 3, 401-418 (1985)

31. T. UEDA, Fatou sets in complex dynamics on projective spaces, J. Math. Soc. Jpn. 46, 545-555 (1994)

32. L. VIVAS, Parametrization of unstable manifolds for parabolic skew-products, preprint 2014, arXiv: 1411.3110 